\title{
ANÁLISIS CUANTITATIVO DEL CONOCIMIENTO TRADICIONAL DE LAS PLANTAS MEDICINALES EN SAN RAFAEL, COXCATLÁN, VALLE DE TEHUACÁN-CUICATLÁN, PUEBLA, MÉXICO
}

\author{
Margarita Canales Martínez ${ }^{1}$, Tzasná Hernández Delgado ${ }^{1}$, Javier \\ Caballero Nieto ${ }^{2}$, Alfonso Romo de Vivar Romo ${ }^{3}$, Ángel Durán Díaz ${ }^{1}$ y \\ RAFAel LiRA SAADE ${ }^{1}$
}

${ }^{1}$ Universidad Nacional Autónoma de México, Facultad de Estudios SuperioresIztacala, Unidad de Biotecnología y Prototipos, Laboratorio de Fitoquímica, 54090 Tlalnepantla, Estado de México, México

${ }^{2}$ Universidad Nacional Autónoma de México, Instituto de Biología, Jardín Botánico, Ciudad Universitaria, Apdo. postal 70-614, 04510 Distrito Federal, México

${ }^{3}$ Universidad Nacional Autónoma de México, Instituto de Química, Ciudad Universitaria, Circuito Exterior, 04510 Distrito Federal, México

\section{RESUMEN}

En este trabajo se presentan los resultados de un estudio sobre la consistencia y distribución del conocimiento tradicional de la flora medicinal de San Rafael, en el municipio de Coxcatlán, Puebla. Se efectuaron dos tipos de entrevistas (abierta y estructurada) a 60 informantes. La aplicación de la prueba de ji cuadrada y el coeficiente de Spearman a los datos, revelaron que no existen diferencias significativas en el conocimiento sobre las plantas medicinales y las características personales de los informantes, aunque pudo observarse una tendencia que señala que las personas de más edad conocen un mayor número de plantas que los jóvenes. Un análisis de correspondencia mostró que no hay ningún patrón de distribución del conocimiento sobre la flora medicinal y que la variación observada es al azar. El análisis de los datos obtenidos en la entrevista estructurada y ajustados a un modelo lineal frente a los datos de la primera entrevista, mostró que más de $90 \%$ de los eventos registrados en la entrevista estructurada son determinados por lo registrado en las entrevistas abiertas. El reducido tamaño del poblado y la sistemática interacción entre sus habitantes, podrían explicar la homogeneidad en el conocimiento tradicional de los pobladores de San Rafael acerca de las propiedades curativas de las plantas.

Palabras clave: conocimiento tradicional, Coxcatlán, etnobotánica, plantas medicinales, Puebla, San Rafael. 


\section{ABSTRACT}

This study was directed to document the consistency and distribution of traditional knowledge concerning the medicinal flora at the village of San Rafael, municipality of Coxcatlán. Two types of interviews (free listing and structured) were applied to 60 informants. Data analysis using chi-square and the Spearman coefficient, revealed no significant differences in knowledge on medicinal plants between gender, age, occupation, provenance and residence time in the village. Nevertheless, it was observed that older people recognized a larger number of plants than the younger ones. Correspondence analysis revealed a nonclear pattern in the distribution of the knowledge of medicinal flora. The analysis of the results obtained from structured interviews and adjusted to a linear model versus the results of the first interviews showed the strength and congruence of the traditional knowledge by the inhabitants of San Rafael about medicinal plants. The interviewed informants recognized more than $90 \%$ of the species they mentioned in the open interviews and also mentioned the same medicinal use. The size of the village and the systematic interactions between its inhabitants, might explain the homogeneity of the traditional knowledge of San Rafael's residents concerning the healing properties of the plants.

Key words: Coxcatlán, ethnobotany, medicinal plants, Puebla, San Rafael, traditional knowledge.

\section{INTRODUCCIÓN}

Las comunidades indígenas poseen un profundo entendimiento de su medio ambiente y su ecología (Leonti et al., 2003; Caballero y Cortés, 2001; Cotton, 1997; Casas et al., 1994). Saben de numerosos usos que se les pueden dar a las plantas -por ejemplo como medicinas- (Ankli et al., 2002; Frei et al., 1998; Heinrich et al., 1998). Estos conocimientos constituyen una base importante para la conservación de la biodiversidad global y para su uso sustentable (Frei et al., 1998; Robineau y Soejarto, 1996).

Las plantas curativas son el recurso terapéutico por excelencia de la medicina tradicional mexicana, que en gran parte es aún rescatable y puede constituir un importante elemento para implementar nuevos planes de salud, que combinen el conocimiento popular con el científico (Argueta y Cano, 1994).

El Valle de Tehuacán-Cuicatlán merece especial atención, porque en esta región se ubica una de las reservas bióticas más relevantes de México. En 10,000 $\mathrm{km}^{2}$ de superficie, probablemente posee la mayor diversidad biológica $(2,700$ 
especies de plantas vasculares) de las zonas áridas de Norteamérica (Casas et al., 2001). En esta zona prospera entre 10 y $11.4 \%$ de la flora mexicana, con 365 especies endémicas que representan $13.9 \%$ del total, mientras que más de $25 \%$ de las plantas vasculares del valle se usa por diferentes comunidades humanas que habitan la región. Por otro lado, el Valle de Tehuacán-Cuicatlán también posee una importante diversidad cultural (Dávila et al., 2002).

San Rafael, es un poblado que pertenece al municipio de Coxcatlán, que se localiza dentro del Valle de Tehuacán-Cuicatlán, en el estado de Puebla. El sitio es objeto de una línea de investigación etnobotánica regional, que pretende conocer la condición actual de los recursos naturales, así como el proceso de cultivo, manipulación y domesticación de plantas, encaminada a proveer herramientas de manejo para el uso y preservación de los recursos naturales dentro de la Reserva de la Biósfera (Lira, 2001). En la comunidad de San Rafael se ha realizado un trabajo sobre la flora útil (Rosas, 2003) y otro sobre la composición, manejo y uso de los vegetales de los huertos (Blanckaert et al., 2004). La presente contribución ha sido la base para realizar un estudio sobre la fitoquímica en relación con el uso tradicional de las plantas medicinales de esta región (Canales et al., 2005).

En el curso de la investigación etnobotánica surge la necesidad de verificar los datos recopilados durante las entrevistas con los informantes, para evitar confusiones al momento de colectar las plantas y documentar el uso preciso que se les da en la región, este es uno de los aspectos más difíciles para la recolección de datos. Se ha visto que las inconsistencias internas pueden ser detectadas cuando a un individuo se le presentan las mismas especies en varias ocasiones (Cotton, 1997). En años recientes, se han publicado algunos estudios cuantitativos sobre la etnobotánica de las plantas medicinales (Alcorn, 1984; Friedman et al., 1986; Moerman, 1999; Frei et al., 1998) pero sólo algunos de ellos (Friedman et al., 1986; Frei et al., 1998) analizan sus datos con respecto a la importancia cultural de las plantas dentro de una cultura específica.

El objetivo de este trabajo fue obtener un inventario de las especies medicinales y documentar la forma de uso que les dan los habitantes de San Rafael. Adicionalmente, se analizó si existía alguna variación del conocimiento sobre las plantas (tanto interna de cada informante, como entre la población) que utilizan como medicinales, es decir se analizó la consistencia y distribución del conocimiento tradicional de la flora medicinal entre la población de San Rafael, con respecto a las características personales de los informantes. 


\section{ÁREA DE ESTUDIO}

San Rafael, municipio de Coxcatlán, Puebla, se localiza al sureste de Tehuacán, en el Valle de Tehuacán-Cuicatlán, entre $18^{\circ} 12^{\prime}$ y $18^{\circ} 14^{\prime}$ de latitud norte, así como $97^{\circ} 07^{\prime}$ y $97^{\circ} 09^{\prime}$ de longitud oeste, en un intervalo altitudinal de 957 a 1400 m (Fig. 1). El clima es de categoría $B_{1}\left(h^{\prime}\right) w^{\prime \prime}(w) e g$ de acuerdo con la clasificación de Koeppen, modificada por García (1981), el cual corresponde a un tipo seco o árido con lluvias en verano y temperatura media anual de $22{ }^{\circ} \mathrm{C}$, variando entre $25^{\circ} \mathrm{C}$ en abril y mayo, y $18{ }^{\circ} \mathrm{C}$ en enero (Medina, 2000). La vegetación predominante es un bosque tropical caducifolio, donde Escontria chiotilla (F. A. C. Weber) Rose y Pachycereus weberi (J. Coulter) Backeb. son particularmente abundantes (Valiente-Banuet et al., 2000).

La comunidad de San Rafael es relativamente de poca antigüedad. Se fundó a principios del siglo XX (Rosas, 2003) y cuenta con 298 habitantes (151 mujeres y 147 hombres) (Anónimo, 2001). La población joven abarca actualmente 22\% del total, este grupo es el más afectado por la emigración, ya que muchos lugareños entre 15 y 22 años abandonan la comunidad en busca de mejores oportunidades educativas (12\% se encuentra en Tehuacán o en la ciudad de Puebla estudiando la licenciatura) y de trabajo (23\% trabajan en Estados Unidos, 35\% laboran en las maquiladoras de Ajalpa, Tehuacán y Zinacantepec) (Anónimo, 2001). La actividad económica más importante es la agricultura, cuyo principal ingreso proviene del cultivo de la caña de azúcar. Los campesinos de la comunidad también se dedican a la cría de ganado caprino, la recolección de frutos, semillas, leña y madera para diversos fines (Rosas, 2003). Esta comunidad únicamente cuenta con una casa de salud, donde una enfermera ofrece servicios muy básicos y vigila el crecimiento de los niños menores de tres años, además de coordinar las campañas de vacunación (Rosas, 2003).

\section{MATERIALES Y MÉTODOS}

Recopilación de la información etnobotánica

Los datos para este trabajo fueron recabados de mayo 2001 a abril de 2002 y se obtuvieron de un total de 60 personas (45 mujeres y 15 hombres).

Se realizaron dos entrevistas a cada informante, la primera de las cuales fue abierta y en ella se tomaron sus datos personales (ocupación, edad, ubicación de su 
Canales et al.: Conocimiento tradicional de plantas medicinales en San Rafael, Coxcatlán, Puebla

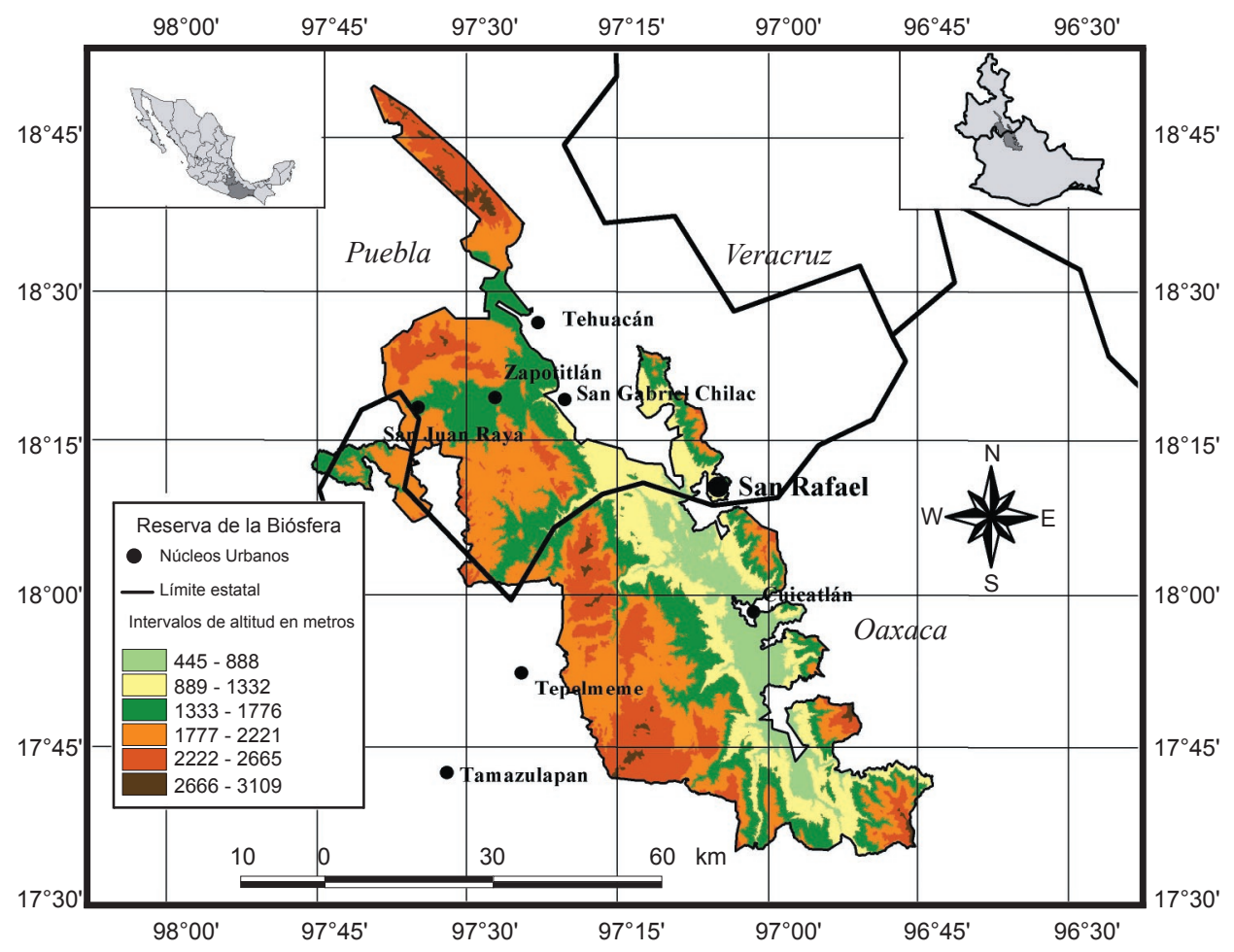

Fig. 1. Ubicación geográfica del poblado de San Rafael, con referencia a la Reserva de la Biósfera Valle de Tehuacán-Cuicatlán.

domicilio, escolaridad, lugar de origen y tiempo viviendo en San Rafael), además de los relativos a las plantas medicinales que usan, a la forma de usarlas y a los padecimientos que tratan con ellas.

Para confirmar y ampliar la información obtenida de esta manera, seis meses después, los mismos informantes fueron sometidos a la segunda entrevista (entrevista estructurada), con la cual se buscaba determinar si reconocían y asignaban el mismo uso a las especies mencionadas por ellos con anterioridad. En este caso, se usó como ayuda visual un catálogo de ejemplares secos y prensados de cada una de las especies medicinales, así como imágenes de las plantas vivas que se les presentaron en una computadora portátil.

Los especímenes usados para la segunda entrevista fueron colectados en el pueblo de San Rafael y sus alrededores. Para ello se hicieron caminatas con algunos de los informantes, con la finalidad de localizar de manera inequívoca las plantas 
en cuestión. Un juego completo de estos materiales se depositó en el herbario de la Facultad de Estudios Superiores de Iztacala (IZTA) y duplicados en el herbario Nacional del Instituto de Biología, Universidad Nacional Autónoma de México (MEXU).

Es importante señalar que las recolecciones se llevaron a cabo con el permiso del Instituto Nacional de Ecología de México y que no se conservó ningún material vivo. Cada visita al poblado se hizo con el consentimiento de las autoridades de San Rafael y los datos etnobotánicos recabados fueron proporcionados voluntariamente por los informantes entrevistados.

Análisis estadísticos

La prueba de ji cuadrada $\left(\mathrm{X}^{2}\right)$ se usó para determinar si existía una relación significativa entre el número de plantas medicinales mencionadas por las personas en la entrevista abierta y las reconocidas (nombre y/o usos) en la entrevista estructurada. La prueba se hizo considerando el género, la edad, el lugar de origen, la ocupación y la ubicación de la vivienda en el poblado de los informantes. Adicionalmente, se calculó el coeficiente de correlación de Spearman para determinar si existía relación entre la edad y el tiempo de residencia de estas personas respecto a la cantidad de plantas medicinales de su cognición.

Se ajustó un modelo logarítmico para definir la relación entre el número de especies mencionadas por los informantes durante la entrevista abierta y el número de especies reconocidas (sólo el nombre) en la entrevista estructurada. Posteriormente, se usó un modelo lineal para determinar la relación entre el número de especies mencionadas en la entrevista abierta y el número de especies reconocidas como medicinales (nombre y forma de uso) en la entrevista estructurada.

Con los datos obtenidos de la entrevista estructurada, se construyó una matriz de caracteres binarios ( 1 el informante reconoce a la planta como medicinal; 0 no la reconoce como medicinal). Con base en esta matriz se hizo un análisis de ordenación por correspondencia, para determinar si existía algún patrón en la distribución del conocimiento sobre la flora medicinal. Es decir, si existía uno o más grupos de personas con determinadas características que conocieran más plantas medicinales. La matriz se formó de 60 columnas (representando a los 60 informantes) y 46 renglones (representando a las especies de plantas medicinales). El análisis se realizó utilizando la versión 2.0 del paquete estadístico NTSYS (Rohlf, 1997). 


\section{RESULTADOS Y DISCUSIÓN}

Informantes y flora medicinal

Las personas entrevistadas proporcionaron información de 46 especies medicinales usadas para el tratamiento de diferentes enfermedades (Apéndice 1), mismas que pertenecen a 28 familias y 46 géneros de plantas vasculares (Apéndice 2). La familia Asteraceae es la que presentó el mayor número de registros, lo cual no es tan sorprendente, pues se trata del grupo mejor representado en la flora de la zona (Rosas, 2003) y concuerda con lo encontrado al respecto por Moerman et al. (1999). De las especies, $47.8 \%$ son silvestres, $28.2 \%$ se cultivan en los huertos, mientras $23.9 \%$ son plantas medicinales que se adquieren en mercados. La alta proporción de plantas silvestres empleadas por la gente de San Rafael, es una muestra de la fuerte dependencia que se tiene en esa comunidad de su entorno vegetal natural para aliviar diversos malestares.

Relación entre el conocimiento y las características de los informantes

Las pruebas de ji cuadrada no arrojaron diferencias significativas ( $p>0.05)$ en ninguno de los casos en que se aplicaron. Esto indica que el número de plantas que conocen las personas de San Rafael, no está relacionado ni con la zona del pueblo donde viven, ni con su género, escolaridad, ocupación o lugar de origen. No obstante, se observan ciertas tendencias en algunos casos. Así, se tiene que las mujeres conocen un mayor número de plantas medicinales, lo cual, de acuerdo con Boster (1985), se puede atribuir a la división que existe usualmente del trabajo intelectual y físico entre hombres y mujeres en comunidades rurales. Este mismo comportamiento fue registrado por Hernández et al. (2003), quienes realizaron un estudio sobre las plantas medicinales utilizadas en la cercana población de Zapotitlán de las Salinas, Puebla, para aliviar enfermedades gastrointestinales. Otra tendencia observada, a pesar de no ser estadísticamente significativa, consistió en que las personas sin escolaridad también conocieron más especies medicinales, lo cual posiblemente se deba a que este grupo de habitantes es el que mantiene una mayor dependencia en el uso de las plantas para la curación de sus padecimientos más comunes.

La no significancia de la relación entre el conocimiento de los informantes y el sitio de ubicación de sus viviendas es fácil de explicar, pues San Rafael es una población muy pequeña, con dos zonas de vivienda separadas únicamente por una 
franja de carretera y cuyos habitantes en muchos casos están emparentados entre sí. Adicionalmente, se observó que la gente de San Rafael realiza muchas actividades conjuntas de mejora para el poblado y esto les da oportunidad de intercambiar ideas y con ello, probablemente, de establecer un flujo de información. Tal hecho podría explicar también la nula significancia del lugar de origen en el conocimiento de los informantes.

Los coeficientes de correlación de Spearman revelaron una correlación baja, aunque estadísticamente significativa, entre el conocimiento de los informantes y su edad, así como el número de años de su residencia en el sitio. Se encontró que las personas de mayor edad $\left(\mathrm{r}=0.55 ; \mathrm{p}=8.0 \times 10^{-6}\right)$ y con mayor antigüedad en el poblado $\left(\mathrm{r}=0.45 ; \mathrm{p}=3.77 \mathrm{X} 10^{-4}\right)$, son quienes conocen más plantas de uso medicinal. En la Figura 2 se observa que entre estas dos variables la tendencia de los puntos es directa. Lo anterior coincide con lo indicado por Garro (1986), quien sugiere que el conocimiento y la experiencia respecto a la enfermedad suelen aumentar con la edad.

El modelo que explicó la relación entre el número de plantas mencionadas en la entrevista abierta y las que reconocían (sólo el nombre) en la entrevista estructurada, fue de tipo $\operatorname{logarítmico~}(\mathrm{y}=14.057 \operatorname{Ln}(\mathrm{x})-6.9898)$ y presentó un coeficiente de determinación de $\mathrm{r}^{2}=0.6177$. Este modelo indica que la cantidad de especies compiladas durante la entrevista estructurada está determinada en $61.77 \%$ por la registrada en la entrevista abierta (Fig. 3).

La relación entre el número de plantas mencionadas en la entrevista abierta y el de las reconocidas como medicinales (nombre y uso) en la entrevista estructurada (Fig. 4), se ajustó a un modelo lineal $(\mathrm{y}=0.862 \mathrm{x}-0.8598)$ con un coeficiente de determinación de $\mathrm{r}^{2}=0.9262$, con lo cual se confirmó que $92.62 \%$ de los eventos recopilados en la entrevista estructurada son determinados por lo registrado en las entrevistas abiertas. Lo anterior confirma la consistencia en el conocimiento que tienen los informantes sobre las plantas medicinales que mencionaron en la primera entrevista. El porcentaje ignorado se debió posiblemente a las características del estímulo, ya que en las primeras entrevistas estructuradas sólo se mostró un catálogo con ejemplares secos y montados. Puesto que se notó que los informantes tenían dificultad para reconocer las plantas preservadas de esta manera, se optó en las siguientes entrevistas por mostrar además su imagen, con lo que se observó que se distinguían fácilmente los ejemplares. Tal experiencia concuerda con lo citado por Boster (1985), quien señala que el estímulo debe ser adecuado y real. Con este modelo, entonces, se confirmó que las personas entrevistadas sí mencionaron en la entrevista abierta las plantas que realmente conocen. 


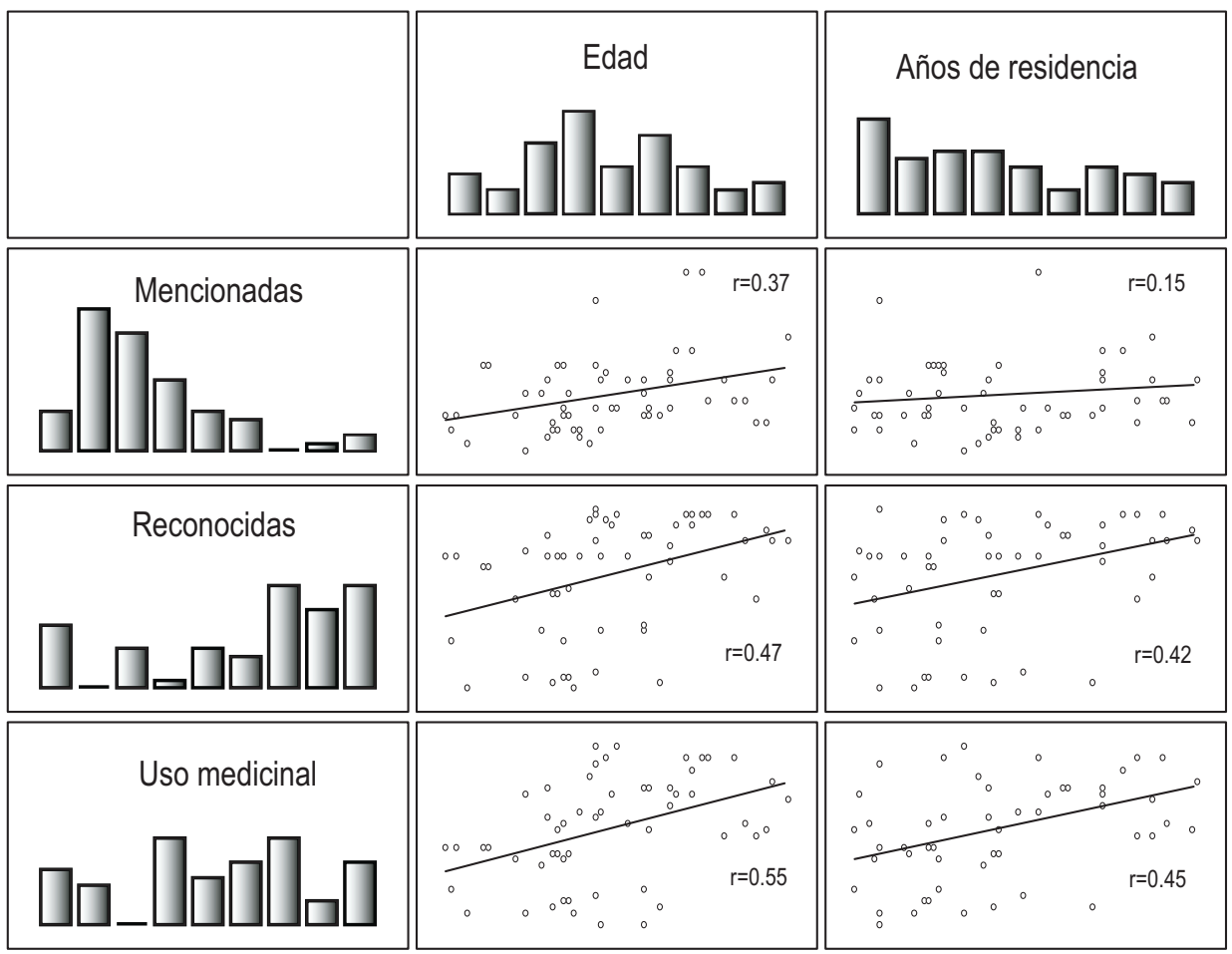

Fig. 2. Relación entre el número de plantas medicinales mencionadas en la entrevista abierta, el número de plantas reconocidas en la entrevista estructurada (sólo el nombre) y el número de plantas reconocidas como medicinales (nombre y uso) en la entrevista estructurada con la edad de los informantes y los años de residencia en San Rafael. Las barras representan los histogramas de cada uno de los grupos de datos correspondientes a cada recuadro.

Con el análisis de ordenación por correspondencia (Fig. 5) se pudo confirmar que no hay ningún patrón de distribución en cuanto al conocimiento que tiene la gente de San Rafael sobre su flora medicinal. Es decir, que no existe ningún grupo de personas que conozca un mayor o menor número de plantas medicinales. En el centro de la gráfica se localizan los informantes que registraron el consenso más alto. En la parte superior se observa que se separan los informantes 31, 47, 58 y 60 , pues fueron los únicos que reconocieron a la chupandilla (Cyrtocarpa procera) y a la biznaga (Ferocactus latispinus) como medicinales, además de referir que utilizan a ambas plantas a la vez en una infusión para aliviar males del riñón. 


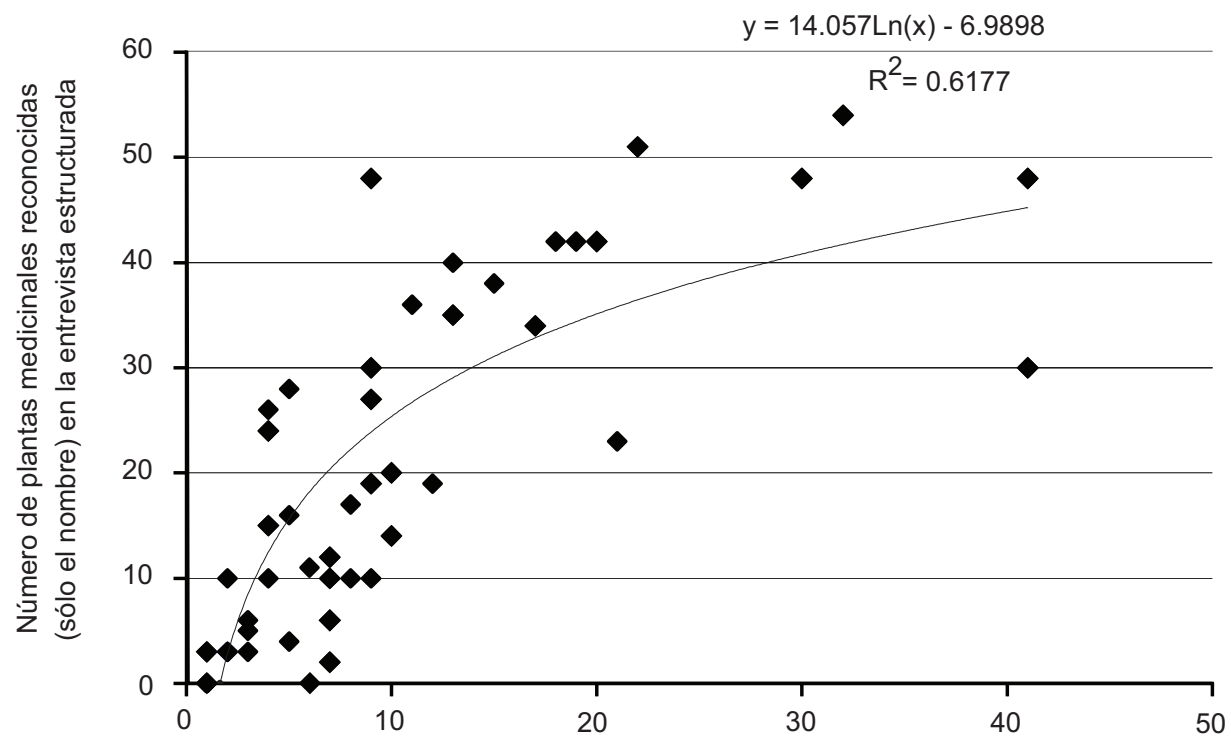

Número de plantas medicinales mencionadas en la entrevista abierta

Fig. 3. Modelo determinado entre el número de especies mencionadas en la entrevista abierta y el número de especies reconocidas (sólo el nombre) en la entrevista estructurada por los informantes.

$$
\begin{gathered}
y=0.862 x-0.8598 \\
R^{2}=0.9262
\end{gathered}
$$
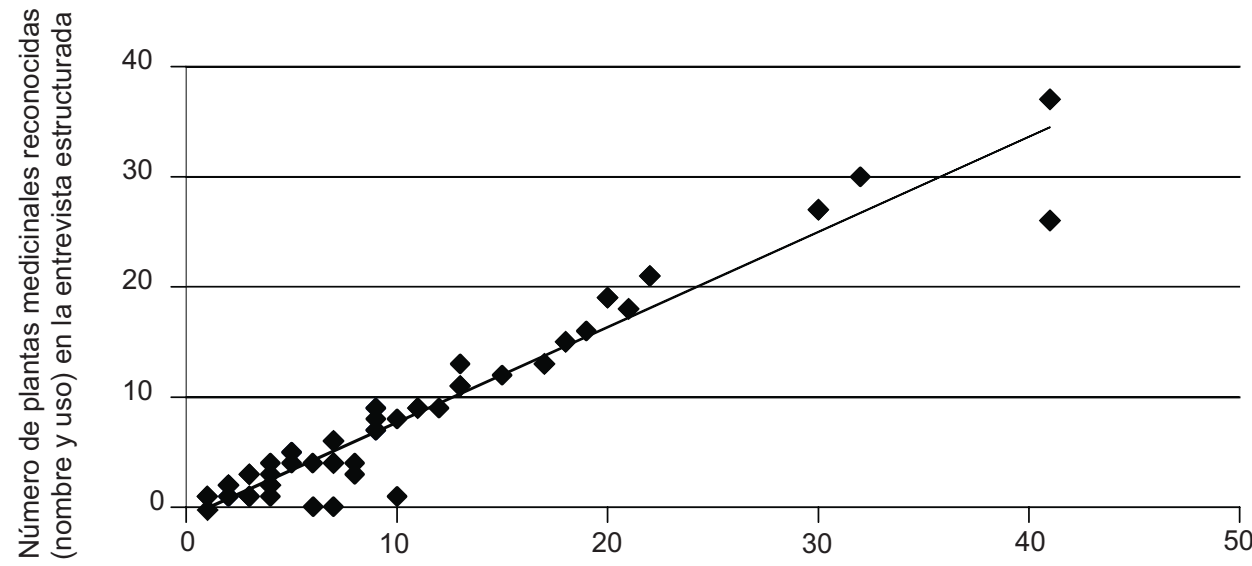

Número de plantas medicinales mencionadas en la entrevista abierta

Fig. 4. Relación entre el número de especies mencionadas en la entrevista abierta y número de especies reconocidas con uso medicinal (nombre y uso) en la entrevista estructurada. 
Canales et al.: Conocimiento tradicional de plantas medicinales en San Rafael, Coxcatlán, Puebla

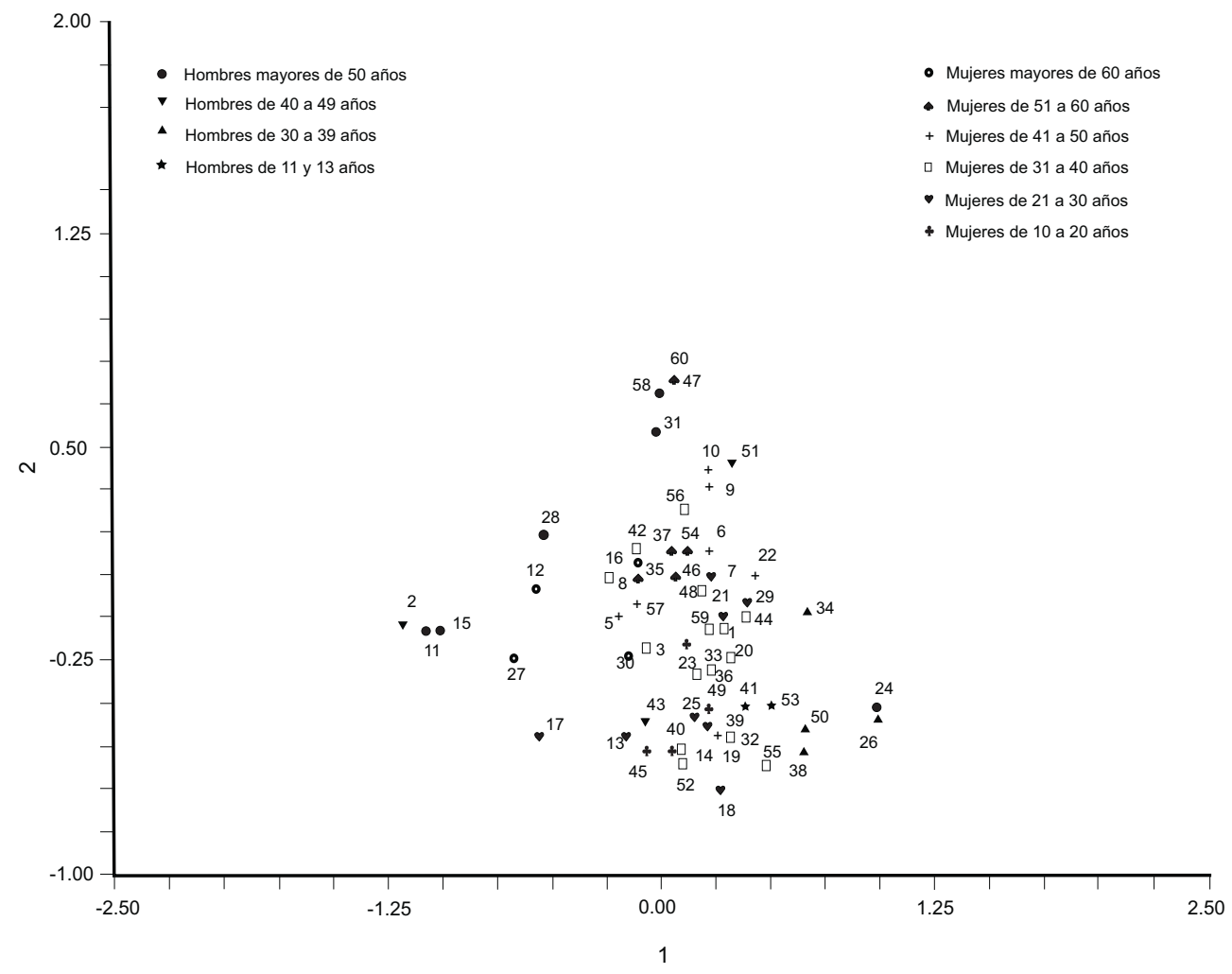

Fig. 5. Análisis de correspondencia de los informantes realizado con los datos de la entrevista estructurada. Los números identifican a cada uno de los informantes.

El hecho de que no haya ningún patrón en cuanto a la distribución del conocimiento entre la población de San Rafael sobre las plantas medicinales, se debe probablemente a que en este poblado existe una institución comunitaria que crea un espacio en el cual el conocimiento se comparte, ya que periódicamente se realizan reuniones a las cuales es obligatorio que asista el representante de cada familia. En estas juntas se exponen y resuelven los problemas más relevantes de la comunidad y dado que son puntos muy importantes los que se tratan, si alguna persona no asiste, se le aplica un castigo que consiste en pasar uno o varios días en la cárcel del pueblo. La junta es organizada y dirigida por el Juez de Paz, quien controla la asistencia de cada miembro representante de familia. Además de estas reuniones, se realizan otras a las que asiste la mayoría de las mujeres responsables de familia y en las que se decide la manera en que se distribuyen los apoyos que aportan diferentes instituciones, principalmente para resolver problemas de salud. Lo anterior, unido al 
hecho de que muchas personas están emparentadas entre sí, propicia que exista una intensa comunicación entre todas las familias de San Rafael.

Otra razón de este compartimiento cultural, consiste en que el pueblo está formado por una comunidad mestiza que ha incorporado dos elementos de conocimiento de medicina herbolaria, uno obtenido de la experimentación sobre elementos de la flora local y el otro relacionado con plantas traídas hace siglos para tal propósito de España, saber que sigue vigente en todo el país.

Las diferencias en el conocimiento que están relacionadas con el género y edad no son tan importantes, parece ser que sólo son debidas a la idiosincrasia. En esta comunidad se ha llevado a cabo una socialización de la información, porque aun cuando el lugar de origen de las personas es diferente y el pueblo está dividido en dos zonas bien marcadas por la franja de carretera, no hay discrepancias significativas en el conocimiento y uso de su flora medicinal.

\section{AGRADECIMIENTOS}

Estamos muy agradecidos con la gente de San Rafael, por la información que nos ofrecieron sobre su flora medicinal, por su amistad y hospitalidad; al Dr. Marco Aurelio Rodríguez M. y a la Biól. Rocío Rosas L. por su gran apoyo en el trabajo de campo; al Dr. Oswaldo Téllez V. por las observaciones realizadas al escrito original; a la Dra. Isabelle Blanckaert por la corrección del resumen en inglés; al Dr. Raymundo Montoya A. por el diseño de la figura del mapa. Esta investigación fue financiada por el Consejo Nacional de Ciencia y Tecnología a través del proyecto CONACYT 400389G35-450, bajo la responsabilidad del último autor durante los años 2001 al 2004.

\section{LITERATURA CITADA}

Alcorn, J. B. 1984. Huastec Mayan ethnobotany. University of Texas Press. Austin, TX. 982 pp.

Ankli, A., M. Heinrich., P. Bork, L. Wolfram, P. Bauerfeind, R. Brun, C. Schmid, C. Weiss, R. Bruggisser, J. Gertsch, M. Wasescha y O. Sticher. 2002. Yucatec Mayan medicinal plants: evaluation based on indigenous uses. J. Ethnopharmacol. 79: 43-52.

Anónimo. Censo de Población 2001. Secretaría de Salud. Casa de salud de la comunidad. Instituto Mexicano del Seguro Social. Ayuntamiento de Coxcatlán, Puebla, México. $10 \mathrm{pp}$. 
Argueta, V. A. y A. J. Cano. 1994. Atlas de las plantas de la medicina tradicional mexicana. Instituto Nacional Indigenista. México, D.F. 1785 pp.

Blanckaert, I., R. L. Swennen, M. Paredes Flores, R. Rosas López y R. Lira Saade. 2004. Floristic composition, plant uses and management practices in homegardens of San Rafael Coxcatlán, Valle de Tehuacán-Cuicatlán, México. J. Arid Environ. 57: 3962.

Boster, J. S. 1985. "Requiem for the omniscient informant": There is life in the old girl yet. In: Dougherty J. W. D. (ed.). Directions in cognitive anthropology. University of Illinois Press. Urbana and Chicago. pp. 177-197.

Caballero, J. y L. Cortés. 2001. Percepción, uso y manejo tradicional de los recursos vegetales en México. In: Rendón, B., S. Rebollar, J. Caballero y M. A. Martínez (eds.). Plantas cultura y sociedad. Universidad Autónoma Metropolitana-Secretaría de Medio Ambiente y Recursos Naturales. México, D.F. pp. 79-100.

Canales M., T. Hernández, J. Caballero, A. Romo de Vivar, G. Ávila, A. Durán y R. Lira. 2005. Informant consensus factor and antibacterial activity of the medicinal plants used by the people of San Rafael Coxcatlán, Puebla. México. J. Ethnopharmacol. 97: 429-439.

Casas, A., A. Valiente-Banuet, J. L. Viveros, P. Dávila, R. Lira, L. Cortés, R. Medina e I. Rodríguez Arévalo. 2001. Plant resources of the Tehuacán-Cuicatlán Valley, México. Econ. Bot. 55(1): 129-166.

Casas, A., J. L. Viveros y J. Caballero. 1994. Etnobotánica mixteca. Sociedad, cultura y recursos naturales en la montaña de Guerrero. Instituto Nacional Indigenista. México, D.F. 366 pp.

Cotton, C. M. 1997. Ethnobotany. Principles and aplications. John Wiley \& Sons. Chichester. $424 \mathrm{pp}$.

Dávila, P., M. C. Arizmendi, A. Valiente-Banuet, J. L. Villaseñor, A. Casas y R. Lira. 2002. Biological diversity in the Tehuacán-Cuicatlán Valley, México. Biodiversity and Conservation 11: 421-442.

Fernández, B. M. N. 1999. Análisis de la dinámica de comunidades vegetales con relación a la evolución del paisaje, en la zona semiárida de Coxcatlán, Puebla. Tesis de maestría. Universidad Nacional Autónoma de México. México, D.F. 115 pp.

Frei, B., M. Baltisberger, O. Sticher y M. Heinrich. 1998. Medical ethnobotany of the Zapotecs of the Isthmus-Sierra (Oaxaca, Mexico): Documentation and assessment of indigenous uses. J. Ethnopharmacol. 62: 149-165.

Friedman, J., Z. Yaniv, A. Dafni y D. Palewitch. 1986. A preliminary classification of the healing potential of medicinal plants, based on a rational analysis of an ethnopharmacological field survey among beduins in the Negev Desert, Israel. J. Ethnopharmacol. 16: 275-287.

García, M. E. 1981. Modificaciones al sistema de clasificación climática de Koppen. 3a. ed. Instituto de Geografía, Universidad Nacional Autónoma de México. México, D.F. $252 \mathrm{pp}$.

Garro, L. C. 1986. Intracultural variation in folk medical knowledge: A comparison between curers and noncurers. Am. Anthropol. 88: 351-370. 
Heinrich, M., Ankli, A., Frei, B. y Weimann, C. 1998. Medicinal plants in Mexico: healers consensus and cultural importance. Soc. Sci. Med. 47: 1859-1871.

Hernández, T., M. Canales, J. G. Ávila, A. Durán, J. Caballero, A. Romo de Vivar y R. Lira. 2003. Ethnobotany and antibacterial activity of some plants used in traditional medicine of Zapotitlán de las Salinas, Puebla (México). J. Ethnopharmacol. 88: 181188.

Leonti, M., O. Sticher y M. Heinrich. 2003. Antiquity of medicinal plant usage in two Macro-Mayan ethnic groups (México). J. Ethnopharmacol. 88: 119-124.

Lira, R. 2001. Los recursos vegetales del Valle de Tehuacán-Cuicatlán desde una perspectiva etnobotánica. Informe técnico. Universidad Nacional Autónoma de México. México, D.F. 9 pp.

Medina, S. J. 2000. Determinación del vigor y estado reproductivo de Stenocereus stellatus (Cactaceae) a lo largo de una cronosecuencia edáfica en un abanico aluvial en Coxcatlán, Valle de Tehuacán. Tesis de licenciatura. Universidad Nacional Autónoma de México. Facultad de Estudios Superiores Iztacala. Tlalnepantla, Méx. 90 pp.

Moerman, D. E., R. W. Pemberton, D. Kiefer y B. Berlin. 1999. A comparative analysis of five medicinal floras. J. Ethnopharmacol. 19: 49-67.

Robineau, L. y D. D. Soejarto. 1996. Tramil: A research project on the medicinal plant resources of the Caribbean. In: Balick, M. J., E. Elisabetsky y S. A. Laird. Medicinal resources of the tropical forests. Columbia University Press. Nueva York. pp. 317325.

Rohlf, F. J. 1997. NTSYS-pc. Numerical taxonomy and multivariate analysis system, version 2.0. Exeter Software. Nueva York.

Rosas, L. R. 2003. Estudio etnobotánico de San Rafael-Coxcatlán. Tesis de licenciatura. Universidad Nacional Autónoma de México. Facultad de Estudios Superiores Iztacala. Tlalnepantla, Méx. 94 pp.

Valiente-Banuet, A., A. Casas, A. Alcántara, P. Dávila, N. Flores-Hernández, M. C. Arismendi, J. Ortega-Ramírez y J. A. Soriano 2000. La vegetación del Valle de Tehuacán-Cuicatlán. Bol. Soc. Bot. Méx. 67: 25-75.

Recibido en abril de 2004. Aceptado en noviembre de 2005. 
Apéndice 1. Datos personales de los informantes entrevistados y número de especies mencionadas y reconocidas. Ubicación del domicilio: N parte nueva; V parte vieja del poblado. Género: M: mujer; H: hombre. Escolaridad: Sin: sin escolaridad; Prim: primaria; Sec: secundaria; Bach: bachillerato; Lic: licenciatura. Ocupación: H: hogar; C: campesino; A: albañil; T: tienda; J: juez; S: casa de salud; E: estudiante. Lugar de origen: SR: San Rafael; Pue: estado

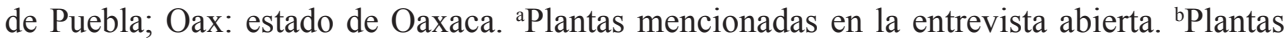

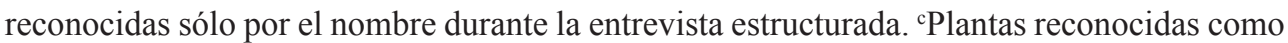
medicinales (nombre y uso) durante la entrevista estructurada.

\begin{tabular}{|c|c|c|c|c|c|c|c|c|c|c|}
\hline & 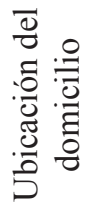 & 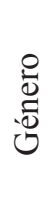 & $\underset{i}{\vec{J}}$ & 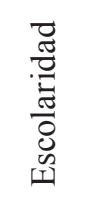 & 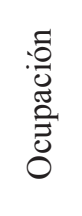 & 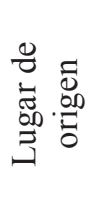 & 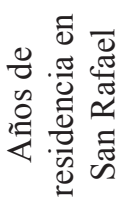 & 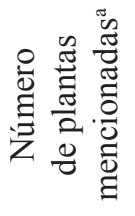 & 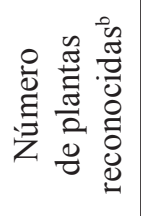 & 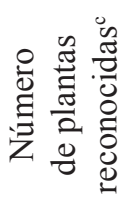 \\
\hline 1 & $\mathrm{~N}$ & M & 36 & Prim & $\mathrm{H}$ & SR & 36 & 3 & 27 & 19 \\
\hline 2 & $\mathrm{~N}$ & $\mathrm{H}$ & 45 & Prim & $\mathrm{C} / \mathrm{J}$ & Pue & 6 & 11 & 27 & 16 \\
\hline 3 & $\mathrm{~N}$ & $\mathrm{M}$ & 39 & Prim & $\mathrm{H}$ & Oax & 21 & 13 & 30 & 18 \\
\hline 4 & V & $\mathrm{H}$ & 30 & Sin & A & Oax & 0.5 & 0 & 0 & 0 \\
\hline 5 & V & $\mathrm{M}$ & 49 & Prim & $\mathrm{H} / \mathrm{T}$ & Pue & 3 & 7 & 23 & 16 \\
\hline 6 & V & $\mathrm{M}$ & 41 & Sin & $\mathrm{H}$ & Oax & 21 & 9 & 35 & 28 \\
\hline 7 & $\mathrm{~V}$ & $\mathrm{M}$ & 26 & Prim & $\mathrm{H}$ & Oax & 4 & 8 & 28 & 22 \\
\hline 8 & V & M & 72 & Sin & $\mathrm{H}$ & SR & 72 & 10 & 30 & 25 \\
\hline 9 & $\mathrm{~N}$ & M & 48 & Prim & $\mathrm{C}$ & Pue & 45 & 5 & 31 & 23 \\
\hline 10 & $\mathrm{~N}$ & $\mathrm{M}$ & 43 & Prim & $\mathrm{H}$ & Pue & 25 & 7 & 35 & 28 \\
\hline 11 & $\mathrm{~N}$ & $\mathrm{M}$ & 50 & Prim & $\mathrm{H}$ & SR & 53 & 10 & 29 & 23 \\
\hline 12 & $\mathrm{~N}$ & $\mathrm{M}$ & 69 & Sin & $\mathrm{H}$ & Pue & 60 & 5 & 19 & 15 \\
\hline 13 & $\mathrm{~N}$ & $\mathrm{M}$ & 24 & $\operatorname{Sin}$ & $\mathrm{H}$ & Pue & 7 & 6 & 19 & 11 \\
\hline 14 & V & $\mathrm{M}$ & 31 & Bach & $\mathrm{H}$ & SR & 31 & 4 & 20 & 12 \\
\hline 15 & $\mathrm{~N}$ & $\mathrm{M}$ & 53 & Prim & $\mathrm{H}$ & SR & 53 & 7 & 29 & 23 \\
\hline 16 & $\mathrm{~N}$ & M & 38 & $\mathrm{Sec}$ & $\mathrm{H} / \mathrm{S}$ & Oax & 28 & 2 & 34 & 25 \\
\hline 17 & $\mathrm{~N}$ & M & 24 & $\operatorname{Sin}$ & $\mathrm{H}$ & Pue & 7 & 5 & 19 & 11 \\
\hline 18 & $\mathrm{~N}$ & $\mathrm{M}$ & 26 & $\mathrm{Sec}$ & $\mathrm{H}$ & Pue & 25 & 1 & 4 & 2 \\
\hline 19 & $\mathrm{~N}$ & $\mathrm{M}$ & 48 & $\operatorname{Sin}$ & $\mathrm{H}$ & Pue & 8 & 10 & 13 & 13 \\
\hline 20 & $\mathrm{~N}$ & $\mathrm{M}$ & 40 & Prim & $\mathrm{H}$ & SR & 40 & 4 & 27 & 19 \\
\hline 21 & $\mathrm{~N}$ & $\mathrm{M}$ & 30 & Prim & $\mathrm{H}$ & SR & 30 & 2 & 27 & 18 \\
\hline 22 & $\mathrm{~N}$ & $\mathrm{M}$ & 42 & Prim & $\mathrm{H}$ & SR & 42 & 6 & 33 & 22 \\
\hline
\end{tabular}


Apéndice 1. Continuación.

\begin{tabular}{|c|c|c|c|c|c|c|c|c|c|c|}
\hline & 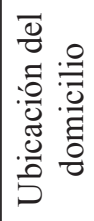 & 过 & $\underset{i}{\tilde{T}}$ & 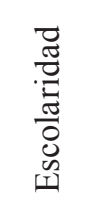 & 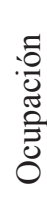 & 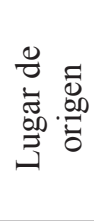 & 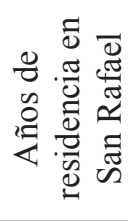 & 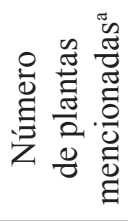 & 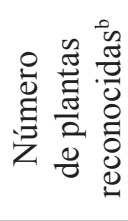 & 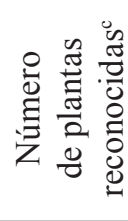 \\
\hline 23 & $\mathrm{~V}$ & M & 15 & Prim & $\mathrm{H}$ & SR & 15 & 2 & 2 & 2 \\
\hline 24 & V & $\mathrm{H}$ & 51 & Prim & $\mathrm{C}$ & SR & 51 & 4 & 3 & 3 \\
\hline 25 & $\mathrm{~V}$ & $\mathrm{M}$ & 29 & Prim & $\mathrm{H}$ & SR & 29 & 8 & 13 & 10 \\
\hline 26 & $\mathrm{~V}$ & $\mathrm{H}$ & 35 & $\mathrm{Sec}$ & $\mathrm{C}$ & Pue & 8 & 3 & 2 & 2 \\
\hline 27 & V & M & 67 & Sin & $\mathrm{H}$ & Oax & 66 & 8 & 30 & 18 \\
\hline 28 & $\mathrm{~V}$ & $\mathrm{H}$ & 71 & Sin & $\mathrm{C}$ & SR & 71 & 5 & 32 & 17 \\
\hline 29 & V & M & 30 & Bach & $\mathrm{H}$ & SR & 30 & 10 & 31 & 23 \\
\hline 30 & V & $\mathrm{M}$ & 75 & Sin & $\mathrm{H}$ & Oax & 63 & 16 & 30 & 21 \\
\hline 31 & V & $\mathrm{H}$ & 65 & Sin & $\mathrm{C}$ & SR & 65 & 8 & 35 & 28 \\
\hline 32 & V & M & 31 & Prim & $\mathrm{H}$ & SR & 31 & 4 & 3 & 3 \\
\hline 33 & V & M & 33 & Sin & $\mathrm{H}$ & Oax & 18 & 6 & 27 & 17 \\
\hline 34 & V & $\mathrm{H}$ & 39 & Prim & $\mathrm{C}$ & Oax & 37 & 6 & 5 & 5 \\
\hline 35 & $\mathrm{~V}$ & $\mathrm{M}$ & 57 & Prim & $\mathrm{H}$ & SR & 57 & 15 & 35 & 26 \\
\hline 36 & V & $\mathrm{M}$ & 34 & Prim & $\mathrm{H}$ & Pue & 14 & 9 & 21 & 12 \\
\hline 37 & V & M & 57 & Prim & $\mathrm{H}$ & Pue & 53 & 14 & 34 & 23 \\
\hline 38 & V & $\mathrm{H}$ & 33 & Prim & $\mathrm{C}$ & Pue & 17 & 5 & 4 & 4 \\
\hline 39 & V & M & 33 & Sin & $\mathrm{H}$ & Pue & 20 & 13 & 11 & 11 \\
\hline 40 & $\mathrm{~N}$ & $\mathrm{M}$ & 19 & $\mathrm{Sec}$ & $\mathrm{H}$ & SR & 19 & 12 & 24 & 14 \\
\hline 41 & $\mathrm{~N}$ & $\mathrm{H}$ & 11 & Prim & $\mathrm{E}$ & Pue & 8 & 6 & 27 & 13 \\
\hline 42 & $\mathrm{~N}$ & M & 39 & Prim & $\mathrm{H}$ & Pue & 8 & 18 & 36 & 27 \\
\hline 43 & $\mathrm{~N}$ & $\mathrm{H}$ & 48 & Prim & $\mathrm{C}$ & Oax & 20 & 9 & 14 & 7 \\
\hline 44 & $\mathrm{~N}$ & $\mathrm{M}$ & 32 & Lic & $\mathrm{H}$ & SR & 32 & 13 & 27 & 16 \\
\hline 45 & V & M & 12 & Prim & $\mathrm{E}$ & Pue & 3 & 4 & 11 & 6 \\
\hline 46 & V & M & 53 & Prim & $\mathrm{H}$ & SR & 53 & 11 & 25 & 19 \\
\hline 47 & V & M & 56 & Prim & $\mathrm{H}$ & Oax & 40 & 26 & 35 & 28 \\
\hline 48 & V & M & 63 & Prim & $\mathrm{H}$ & SR & 63 & 11 & 23 & 15 \\
\hline 49 & $\mathrm{~N}$ & M & 18 & $\mathrm{Sec}$ & $\mathrm{H}$ & SR & 18 & 13 & 25 & 14 \\
\hline 50 & $\mathrm{~V}$ & $\mathrm{H}$ & 34 & Prim & $\mathrm{C}$ & Pue & 18 & 6 & 4 & 4 \\
\hline
\end{tabular}


Apéndice 1. Continuación.

\begin{tabular}{|c|c|c|c|c|c|c|c|c|c|c|}
\hline & 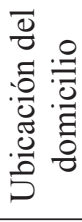 & : & 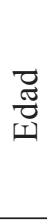 & 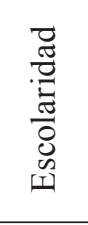 & 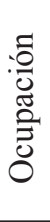 & 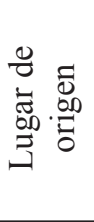 & 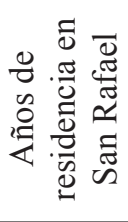 & 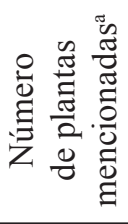 & 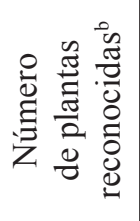 & 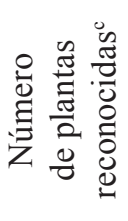 \\
\hline 51 & $\mathrm{~N}$ & $\mathrm{H}$ & 49 & Prim & $\mathrm{C}$ & Pue & 46 & 5 & 31 & 25 \\
\hline 52 & V & M & 32 & Prim & $\mathrm{H}$ & SR & 32 & 4 & 20 & 12 \\
\hline 53 & $\mathrm{~N}$ & $\mathrm{H}$ & 13 & Prim & $\mathrm{E}$ & SR & 13 & 9 & 27 & 10 \\
\hline 54 & V & M & 54 & Prim & $\mathrm{H}$ & Pue & 53 & 16 & 33 & 22 \\
\hline 55 & $\mathrm{~N}$ & M & 40 & Sin & $\mathrm{H}$ & Pue & 8 & 11 & 14 & 14 \\
\hline 56 & $\mathrm{~N}$ & M & 39 & Prim & $\mathrm{H}$ & Pue & 25 & 7 & 35 & 28 \\
\hline 57 & V & M & 49 & Prim & $\mathrm{H}$ & Pue & 3 & 7 & 23 & 16 \\
\hline 58 & V & $\mathrm{H}$ & 60 & Sin & $\mathrm{C}$ & SR & 60 & 7 & 35 & 28 \\
\hline 59 & $\mathrm{~N}$ & M & 36 & Prim & $\mathrm{H}$ & SR & 36 & 4 & 27 & 19 \\
\hline 60 & V & M & 59 & Prim & $\mathrm{H}$ & Oax & 40 & 26 & 35 & 27 \\
\hline
\end{tabular}


Apéndice 2. Especies usadas en la medicina tradicional de San Rafael. aPlantas silvestres

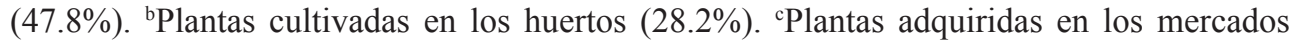
(23.9\%). Parte usada: C: corteza; F: flor; FR: fruto; H: hojas; L: látex; PA: parte aérea; R: raíz.

\begin{tabular}{|c|c|c|c|}
\hline $\begin{array}{l}\text { Familia } \\
\text { Especie } \\
\text { Nombre común }\end{array}$ & $\begin{array}{l}\text { Usos y } \\
\text { aplicaciones para }\end{array}$ & $\begin{array}{l}\text { Parte } \\
\text { usada }\end{array}$ & Forma de uso \\
\hline $\begin{array}{l}\text { Acanthaceae } \\
\text { Gypsacanthus nelsonii E. } \\
\text { J. Lott, V. Jaram. \& Rzed. } \\
\text { Hierba de la tiricia }^{\text {a }}\end{array}$ & Tiricia & $\mathrm{PA}$ & $\begin{array}{l}\text { Se adorna la planta con papel } \\
\text { y globos rojos, se lleva al niño } \\
\text { con tiricia y se le hace bailar y } \\
\text { abrazar a la planta }\end{array}$ \\
\hline $\begin{array}{l}\text { Agavaceae } \\
\text { Agave stricta Salm-Dyck } \\
\text { Magueicillo }^{\mathrm{a}}\end{array}$ & $\begin{array}{l}\text { Heridas } \\
\text { sangrantes }\end{array}$ & PA & $\begin{array}{l}\text { Se corta la penca y la pulpa se } \\
\text { coloca sobre la herida }\end{array}$ \\
\hline $\begin{array}{l}\text { Amaryllidaceae } \\
\text { Aloe vera (L.) Burm. f. } \\
\text { Sábila }^{\text {b }}\end{array}$ & $\begin{array}{l}\text { Irritación en los } \\
\text { ojos, quemadas, } \\
\text { torceduras, } \\
\text { heridas, tos, } \\
\text { anginas } \\
\text { Diabetes }\end{array}$ & PA & $\begin{array}{l}\text { Con la pulpa se hace un licuado } \\
\text { y se toma en ayunas }\end{array}$ \\
\hline $\begin{array}{l}\text { Anacardiaceae } \\
\text { Schinus molle L. } \\
\text { Coabino o pirul }\end{array}$ & $\begin{array}{l}\text { Baño para } \\
\text { parturientas } \\
\text { "Aire" }\end{array}$ & PA & $\begin{array}{l}\text { Se pone a hervir con el agua con } \\
\text { que se va a bañar a la señora } \\
\text { Se pone una ramita bajo la ropa } \\
\text { o atrás de la oreja }\end{array}$ \\
\hline $\begin{array}{l}\text { Cyrtocarpa procera } \\
\text { Kunth } \\
\text { Chupandilla }^{\mathrm{a}}\end{array}$ & Dolor de riñones & $\mathrm{C}$ & $\begin{array}{l}\text { Se prepara un té con la corteza } \\
\text { de la chupandilla combinada } \\
\text { con la biznaga y el cuachalala, } \\
\text { se toma como agua de tiempo }\end{array}$ \\
\hline $\begin{array}{l}\text { Asclepiadaceae } \\
\text { Asclepias linaria Cav. } \\
\text { Romero de monte }^{c}\end{array}$ & $\begin{array}{l}\text { Baño para } \\
\text { parturientas } \\
\text { Cólicos, espanto }\end{array}$ & PA & $\begin{array}{l}\text { Se pone a hervir en el agua del } \\
\text { baño de las señoras } \\
\text { Té, tomado }\end{array}$ \\
\hline $\begin{array}{l}\text { Asteraceae } \\
\text { Artemisia absinthium L. } \\
\text { Hierba maestra }^{\mathrm{c}}\end{array}$ & $\begin{array}{l}\text { Dolor de estó- } \\
\text { mago, dolor en } \\
\text { parturientas, } \\
\text { cólicos, bilis }\end{array}$ & PA & $\begin{array}{l}\text { Té, se toma una tacita en } \\
\text { ayunas }\end{array}$ \\
\hline
\end{tabular}


Apéndice 2. Continuación.

\begin{tabular}{|c|c|c|c|}
\hline $\begin{array}{l}\text { Familia } \\
\text { Especie } \\
\text { Nombre común }\end{array}$ & $\begin{array}{c}\text { Usos y } \\
\text { aplicaciones para }\end{array}$ & $\begin{array}{l}\text { Parte } \\
\text { usada }\end{array}$ & Forma de uso \\
\hline $\begin{array}{l}\text { Flaveria trinervia } \\
\text { (Spreng.) C. Mohr } \\
\text { Hierba del sapo }\end{array}$ & $\begin{array}{l}\text { Disentería, } \\
\text { gastritis } \\
\text { Infección de } \\
\text { heridas }\end{array}$ & $\mathrm{PA}$ & $\begin{array}{l}\text { Té, se toma como agua de } \\
\text { tiempo } \\
\text { Té, se lava la herida infectada }\end{array}$ \\
\hline $\begin{array}{l}\text { Gymnosperma } \\
\text { glutinosum (Spreng.) } \\
\text { Less. } \\
\text { Popote }^{\text {a }}\end{array}$ & Diarrea & $\mathrm{PA}$ & Té, tomado \\
\hline $\begin{array}{l}\text { Matricaria recutita L. } \\
\text { Manzanilla }^{c}\end{array}$ & $\begin{array}{l}\text { Diarrea, alfericia, } \\
\text { garganta, gripa } \\
\text { Irritación en los } \\
\text { ojos }\end{array}$ & PA & $\begin{array}{l}\text { Té, como agua de tiempo } \\
\text { Té, se lavan los ojos }\end{array}$ \\
\hline $\begin{array}{l}\text { Montanoa tomentosa } \\
\text { Cerv. } \\
\text { Acahuite }^{\mathrm{a}}\end{array}$ & Bebés enlechados & PA & $\begin{array}{l}\text { Los retoños de las hojas se } \\
\text { ponen en un cascarón con } \\
\text { leche materna, se acercan a las } \\
\text { brazas hasta que hierva y se les } \\
\text { da a los bebés. Para un niño se } \\
\text { usa leche de la mamá de una } \\
\text { niña y viceversa }\end{array}$ \\
\hline $\begin{array}{l}\text { Tanacetum parthenium } \\
\text { (L.) Sch. Bip. } \\
\text { Santa María }^{c}\end{array}$ & $\begin{array}{l}\text { Baño para } \\
\text { parturientas } \\
\text { Diarrea, coraje }\end{array}$ & $\mathrm{PA}$ & $\begin{array}{l}\text { Se pone a hervir en el agua de } \\
\text { su baño } \\
\text { Té, se toma como agua de } \\
\text { tiempo }\end{array}$ \\
\hline $\begin{array}{l}\text { Verbesina crocata } \\
\text { (Cav.) Less. } \\
\text { Árnica }^{\text {b }}\end{array}$ & $\begin{array}{l}\text { Parturientas, para } \\
\text { restaurar la cadera } \\
\text { Infección de } \\
\text { heridas, ronchas, } \\
\text { quemaduras } \\
\text { Garganta irritada }\end{array}$ & PA & $\begin{array}{l}\text { Se pone a hervir con el agua de } \\
\text { su baño } \\
\text { Té, se lava la parte afectada } \\
\text { Té, se hacen gárgaras }\end{array}$ \\
\hline
\end{tabular}


Apéndice 2. Continuación.

\begin{tabular}{|c|c|c|c|}
\hline $\begin{array}{l}\text { Familia } \\
\text { Especie } \\
\text { Nombre común }\end{array}$ & $\begin{array}{l}\text { Usos y } \\
\text { aplicaciones para }\end{array}$ & $\begin{array}{l}\text { Parte } \\
\text { usada }\end{array}$ & Forma de uso \\
\hline $\begin{array}{l}\text { Viguiera dentata (Cav.) } \\
\text { Sprengel } \\
\text { Chimalacate }^{\mathrm{a}}\end{array}$ & $\begin{array}{l}\text { Parturientas, } \\
\text { chincual } \\
\text { Piquetes de } \\
\text { hormiga }\end{array}$ & PA & $\begin{array}{l}\text { Se pone a hervir con el agua de } \\
\text { su baño } \\
\text { Se restriega una hoja directa- } \\
\text { mente en el piquete }\end{array}$ \\
\hline $\begin{array}{l}\text { Bombacaceae } \\
\text { Ceiba parvifolia } \text { Rose } \\
\text { Pochote }^{\mathrm{a}}\end{array}$ & 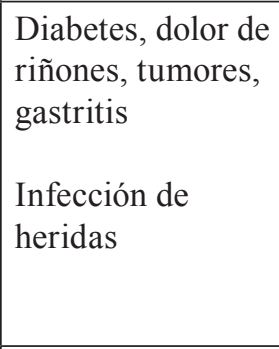 & $\mathrm{C}$ & $\begin{array}{l}\text { Té, se toma como agua de } \\
\text { tiempo } \\
\text { Té, se lavan las heridas infecta- } \\
\text { das; también se pulveriza la cor- } \\
\text { teza seca y se coloca ese polvo } \\
\text { directo sobre la herida }\end{array}$ \\
\hline $\begin{array}{l}\text { Boraginaceae } \\
\text { Borago officinalis } L \text {. } \\
\text { Borrajac }^{c}\end{array}$ & $\begin{array}{l}\text { Tos, garganta } \\
\text { irritada, gripe }\end{array}$ & PA & $\begin{array}{l}\text { Té con leche, se toma muy dulce } \\
\text { de preferencia por la noche }\end{array}$ \\
\hline $\begin{array}{l}\text { Burseraceae } \\
\text { Bursera arida (Rose) } \\
\text { Standley } \\
\text { Aceitillo }^{\text {a }}\end{array}$ & $\begin{array}{l}\text { Infección de una } \\
\text { herida, fuegos, } \\
\text { algodoncillo, } \\
\text { granos en la piel }\end{array}$ & $\mathrm{L}$ & $\begin{array}{l}\text { Se hace un corte al tronco de } \\
\text { la planta y "el juguito" que } \\
\text { escurre, se coloca directo sobre } \\
\text { la parte afectada }\end{array}$ \\
\hline $\begin{array}{l}\text { Cactaceae } \\
\text { Hylocereus undatus } \\
\text { (Haw.) Britton \& Rose } \text { Pitahaya }^{b}\end{array}$ & Gripe & PA & $\begin{array}{l}\text { Se prepara un té y se toma como } \\
\text { agua de tiempo }\end{array}$ \\
\hline $\begin{array}{l}\text { Ferocactus latispinus } \\
\text { (Haw.) Britton \& Rose } \\
\text { Biznaga }^{\text {a }}\end{array}$ & Dolor de riñones & $\mathrm{PA}$ & $\begin{array}{l}\text { Té, combinado con la corteza } \\
\text { de chupandilla y cuachalala. Se } \\
\text { toma como agua de tiempo }\end{array}$ \\
\hline $\begin{array}{l}\text { Opuntia sp. } \\
\text { Nopal }^{\text {b }}\end{array}$ & Diabetes & $\mathrm{PA}$ & Licuado en ayunas \\
\hline $\begin{array}{l}\text { Chenopodiaceae } \\
\text { Chenopodium murale L. } \\
\text { Chaguaquelite }^{\mathrm{a}}\end{array}$ & Alforra & $\mathrm{PA}$ & $\begin{array}{l}\text { Se pone a hervir la planta y se } \\
\text { hace una bolita que se coloca en } \\
\text { el ano del bebé }\end{array}$ \\
\hline
\end{tabular}


Apéndice 2. Continuación.

\begin{tabular}{|c|c|c|c|}
\hline $\begin{array}{l}\text { Familia } \\
\text { Especie } \\
\text { Nombre común }\end{array}$ & $\begin{array}{l}\text { Usos y } \\
\text { aplicaciones para }\end{array}$ & $\begin{array}{l}\text { Parte } \\
\text { usada }\end{array}$ & Forma de uso \\
\hline $\begin{array}{l}\text { Cyperaceae } \\
\text { Cyperus hermaphroditus } \\
\text { (Jacq.) Standl. } \\
\text { Piomía }^{\text {a }}\end{array}$ & $\begin{array}{l}\text { Cólicos } \\
\text { Alfericia }\end{array}$ & $\mathrm{R}$ & $\begin{array}{l}\text { Té, como agua de tiempo } \\
\text { Se esculpe una cruz con la } \\
\text { raíz y se cuelga al cuello del } \\
\text { niño con alfericia }\end{array}$ \\
\hline $\begin{array}{l}\text { Equisetaceae } \\
\text { Equisetum hyemale L. } \\
\text { Cola de iguana o Cola de } \\
\text { caballo }^{\mathrm{c}}\end{array}$ & Dolor de riñones & $\mathrm{PA}$ & $\begin{array}{l}\text { Té, se toma como agua de } \\
\text { tiempo }\end{array}$ \\
\hline $\begin{array}{l}\text { Euphorbiaceae } \\
\text { Acalypha hederacea Torr. } \\
\text { Hierba del pastor }{ }^{\mathrm{a}}\end{array}$ & $\begin{array}{l}\text { Baño para } \\
\text { parturientas } \\
\text { Granos, infección de } \\
\text { heridas, inflamación }\end{array}$ & PA & $\begin{array}{l}\text { Se usa en el baño de las } \\
\text { señoras } \\
\text { Té, se lava y se ponen fomen- } \\
\text { tos en la parte afectada }\end{array}$ \\
\hline $\begin{array}{l}\text { Cnidoscolus chayamansa } \\
\text { McVaugh } \\
\text { Chaya }^{b}\end{array}$ & $\begin{array}{l}\text { Diabetes, } \\
\text { inflamación }\end{array}$ & $\mathrm{H}$ & $\begin{array}{l}\text { Té, tomado como agua de } \\
\text { tiempo }\end{array}$ \\
\hline $\begin{array}{l}\text { Jatropha neopauciflora } \\
\text { Pax } \\
\text { Sangre de grado }^{\mathrm{a}}\end{array}$ & $\begin{array}{l}\text { Fuegos bucales, } \\
\text { infección de heridas, } \\
\text { dolor de dientes }\end{array}$ & $\mathrm{L}$ & $\begin{array}{l}\text { Se hace una herida a la planta } \\
\text { y el látex se pone directamente } \\
\text { sobre la parte afectada }\end{array}$ \\
\hline $\begin{array}{l}\text { Euphorbiaceae } \\
\text { Ricinus communis L. } \\
\text { Higuerilla }^{\mathrm{a}}\end{array}$ & $\begin{array}{l}\text { Pecho, empacho, } \\
\text { calentura }\end{array}$ & $\mathrm{H}$ & $\begin{array}{l}\text { Se unta la espalda y abdomen } \\
\text { con manteca y luego se coloca } \\
\text { una hoja que cubra toda la } \\
\text { región }\end{array}$ \\
\hline $\begin{array}{l}\text { Julianiaceae } \\
\text { Juliania adstringens } \\
\text { (Schldl.) Schldl. } \\
\text { Cuachalala }^{\text {a }}\end{array}$ & $\begin{array}{l}\text { Diabetes, dolor de } \\
\text { riñones, tumores, } \\
\text { gastritis. } \\
\text { Infección de heridas }\end{array}$ & $\mathrm{C}$ & $\begin{array}{l}\text { Té, se toma como agua de } \\
\text { tiempo } \\
\text { Té, se lavan las heridas in- } \\
\text { fectadas; también se pulveriza } \\
\text { la corteza seca y se coloca ese } \\
\text { polvo directo sobre la herida }\end{array}$ \\
\hline
\end{tabular}


Apéndice 2. Continuación.

\begin{tabular}{|c|c|c|c|}
\hline $\begin{array}{l}\text { Familia } \\
\text { Especie } \\
\text { Nombre común } \\
\end{array}$ & $\begin{array}{c}\text { Usos y } \\
\text { aplicaciones para }\end{array}$ & $\begin{array}{l}\text { Parte } \\
\text { usada }\end{array}$ & Forma de uso \\
\hline $\begin{array}{l}\text { Lamiaceae } \\
\text { Mentha } \mathrm{x} \text { piperita } \mathrm{L} . \\
\text { Hierbabuena }^{\mathrm{b}}\end{array}$ & $\begin{array}{l}\text { Estómago, parási- } \\
\text { tos gastrointesti- } \\
\text { nales, gripe }\end{array}$ & PA & Té, como agua de tiempo \\
\hline $\begin{array}{l}\text { Marrubium vulgare L. } \\
\text { Marrubio }^{c}\end{array}$ & $\begin{array}{l}\text { Diarrea, berrinche, } \\
\text { dolor } \\
\text { Infección de una } \\
\text { herida }\end{array}$ & PA & $\begin{array}{l}\text { Té, en ayunas } \\
\text { La herida se lava con el té }\end{array}$ \\
\hline $\begin{array}{l}\text { Ocimum basilicum L. } \\
\text { Albahácar }^{\mathrm{b}}\end{array}$ & $\begin{array}{l}\text { Garganta, parási- } \\
\text { tos, diarrea, dolor } \\
\text { de estómago } \\
\text { Aire }\end{array}$ & PA & $\begin{array}{l}\text { Se prepara un té y se toma lo } \\
\text { más seguido posible } \\
\text { Se pone una ramita bajo la ropa } \\
\text { a la altura del pecho }\end{array}$ \\
\hline \begin{tabular}{|l|} 
Mimosaceae \\
Mimosa luisana \\
Brandegee \\
Uña de gato $^{\mathrm{a}}$ \\
\end{tabular} & Diabetes & PA & Té, como agua de tiempo \\
\hline $\begin{array}{l}\text { Prosopis laevigata } \\
\text { (Humb. \& Bonpl. ex } \\
\text { Willd.) M. C. Johnston } \\
\text { Mezquite }^{\text {b }}\end{array}$ & $\begin{array}{l}\text { Parturientas } \\
\text { Empacho } \\
\text { Irritación de ojos }\end{array}$ & PA & $\begin{array}{l}\text { Se pone a hervir con el agua de } \\
\text { su baño } \\
\text { Té con los retoños y se toma } \\
\text { Té, se toma y se lavan los ojos }\end{array}$ \\
\hline $\begin{array}{l}\text { Myrtaceae } \\
\text { Eucalyptus globulus } \\
\text { Labill. } \\
\text { Eucalipto }^{c} \\
\end{array}$ & Tos & PA & Té, se toma \\
\hline $\begin{array}{l}\text { Psidium guajava L. } \\
\text { Guayabo }^{\mathrm{b}}\end{array}$ & Diarrea, disentería & $\mathrm{H}$ & Té, como agua de tiempo \\
\hline $\begin{array}{l}\text { Nyctaginaceae } \\
\text { Bougainvillea spectabilis } \\
\text { Willd. } \\
\text { Bugambilia }^{\mathrm{b}}\end{array}$ & $\begin{array}{l}\text { Tos y gripa } \\
\text { Tristeza de niños }\end{array}$ & $\mathrm{F}$ & $\begin{array}{l}\text { Té, con la flor morada, se toma a } \\
\text { cualquier hora del día } \\
\text { Las flores rojas las avienta el } \\
\text { niño al apancle y al irse con el } \\
\text { agua las flores, también se va la } \\
\text { tristeza }\end{array}$ \\
\hline
\end{tabular}


Apéndice 2. Continuación.

\begin{tabular}{|c|c|c|c|}
\hline $\begin{array}{l}\text { Familia } \\
\text { Especie } \\
\text { Nombre común }\end{array}$ & $\begin{array}{l}\text { Usos y } \\
\text { aplicaciones para }\end{array}$ & $\begin{array}{l}\text { Parte } \\
\text { usada }\end{array}$ & Forma de uso \\
\hline $\begin{array}{l}\text { Papaveraceae } \\
\text { Argemone mexicana } \mathrm{L} \text {. } \\
\text { Chicalote }^{\mathrm{a}}\end{array}$ & Perrillas & $\mathrm{L}$ & $\begin{array}{l}\text { Se corta una rama y se coloca } \\
\text { "el juguito" directamente en } \\
\text { el ojo }\end{array}$ \\
\hline $\begin{array}{l}\text { Piperaceae } \\
\text { Piper auritum Kunth } \\
\text { Hoja santa }^{\text {b }}\end{array}$ & Abortivo & $\mathrm{H}$ & Té, en ayunas \\
\hline $\begin{array}{l}\text { Rosaceae } \\
\text { Rosa centifolia } \mathrm{L} . \\
\text { Rosa de Castilla }^{\mathrm{c}}\end{array}$ & $\begin{array}{l}\text { Irritación en los } \\
\text { ojos, granos en la } \\
\text { cara, cólico }\end{array}$ & $\mathrm{F}$ & $\begin{array}{l}\text { Té, tomado y se lava la parte } \\
\text { afectada }\end{array}$ \\
\hline $\begin{array}{l}\text { Rutaceae } \\
\text { Ruta chalepensis L. } \\
\text { Ruda }^{\mathrm{b}}\end{array}$ & $\begin{array}{l}\text { Diarrea, garganta, } \\
\text { cólicos, coraje } \\
\text { Tiricia, aire, dolor } \\
\text { de cabeza }\end{array}$ & $\mathrm{PA}$ & $\begin{array}{l}\text { Té, se puede tomar en ayunas } \\
\text { Se pone una ramita tras la } \\
\text { oreja }\end{array}$ \\
\hline $\begin{array}{l}\text { Selaginellaceae } \\
\text { Selaginella lepidophylla } \\
\text { (Hook. \& Grev.) Spring } \\
\text { Siempre viva o doradilla }^{\text {a }}\end{array}$ & Dolor de riñones & PA & Té, como agua de tiempo \\
\hline $\begin{array}{l}\text { Simaroubaceae } \\
\text { Castela tortuosa Liebm. } \\
\text { Venenillo }^{\mathrm{a}}\end{array}$ & $\begin{array}{l}\text { Diabetes, } \\
\text { berrinche, } \\
\text { problemas de } \\
\text { presión arterial }\end{array}$ & PA & Té, como agua de tiempo \\
\hline $\begin{array}{l}\text { Solanaceae } \\
\text { Margaranthus } \\
\text { solanaceus Schltdl. } \\
\text { Totomachea }\end{array}$ & $\begin{array}{l}\text { Diabetes, bilis } \\
\text { Berrinche }\end{array}$ & $\mathrm{H}$ & $\begin{array}{l}\text { Té, en ayunas } \\
\text { A los niños berrinchudos se } \\
\text { les da una hoja a masticar }\end{array}$ \\
\hline $\begin{array}{l}\text { Physalis } \mathrm{sp} . \\
\text { Tomate verde }^{\mathrm{c}}\end{array}$ & Garganta & FR & $\begin{array}{l}\text { Se asan y se colocan calientes } \\
\text { como cataplasma sobre la } \\
\text { garganta }\end{array}$ \\
\hline $\begin{array}{l}\text { Turneraceae } \\
\text { Turnera diffusa Willd. } \\
\text { Ítamo real }^{\mathrm{c}}\end{array}$ & Diarrea, cólicos & $\mathrm{PA}$ & Té, como agua de tiempo \\
\hline $\begin{array}{l}\text { Verbenaceae } \\
\text { Lippia graveolens Kunth } \\
\text { Orégano }^{\mathrm{a}}\end{array}$ & $\begin{array}{l}\text { Diarrea, pecho, } \\
\text { abortivo, dolor de } \\
\text { estómago, cólicos }\end{array}$ & PA & $\begin{array}{l}\text { Té, se toma en ayunas y } \\
\text { durante el día }\end{array}$ \\
\hline
\end{tabular}

